\title{
MCMV triggers ROS/NLRP3-associated inflammasome activation in the inner ear of mice and cultured spiral ganglion neurons, contributing to sensorineural hearing loss
}

\author{
WEI ZHUANG ${ }^{1 *}$, CAIJI WANG ${ }^{2 *}$, XI SHI $^{2 *}$, SHIWEI QIU ${ }^{1}$, SHILI ZHANG ${ }^{2}$, BING XU $^{1}$, MIN CHEN $^{1}$, \\ WEN JIANG ${ }^{2}$, HONGYAN DONG ${ }^{3}$ and YUEHUA QIAO ${ }^{1,2}$ \\ ${ }^{1}$ Clinical Hearing Center, Affiliated Hospital of Xuzhou Medical University, Xuzhou, Jiangsu 221006; \\ ${ }^{2}$ Institute of Audiology and Balance Science, and ${ }^{3}$ Research Facility Center for Morphology, \\ Xuzhou Medical University, Xuzhou, Jiangsu 221004, P.R. China \\ Received April 13, 2017; Accepted February 28, 2018
}

DOI: $10.3892 /$ ijmm.2018.3539

\begin{abstract}
Congenital cytomegalovirus (CMV) infection is the most common infectious cause of sensorineural hearing loss in children. While the importance of CMV-induced SNHL has been described, the mechanisms underlying its pathogenesis and the role of inflammatory responses remain elusive. The present study established an experimental model of hearing loss after systemic infection with murine CMV (MCMV) in newborn mice. Auditory brainstem responses were tested to evaluate hearing at 3 weeks, expression of inflammasomeassociated factors was assessed by immunofluorescence, western blot analysis, reverse transcription-quantitative polymerase chain reaction and ELISA. MCMV sequentially induced inflammasome-associated factors. Furthermore, the inflammasome-associated factors were also increased in cultured spiral ganglion neurons infected with MCMV for $24 \mathrm{~h}$. In addition, MCMV increased the content of reactive oxygen species (ROS). These results suggest that hearing loss caused by MCMV infection may be associated with ROS-induced inflammation.
\end{abstract}

Correspondence to: Professor Hongyan Dong, Research Facility Center for Morphology, Xuzhou Medical University, 209 Tongshan Road, Xuzhou, Jiangsu 221004, P.R. China

E-mail: dhy@xzmc.edu.cn

Professor Yuehua Qiao, Clinical Hearing Center, Affiliated Hospital of Xuzhou Medical University, 99 Huaihai Road, Xuzhou, Jiangsu 221006, P.R. China

E-mail: yuehuaqiao001@163.com

*Contributed equally

Key words: murine congenital cytomegalovirus, sensorineural hearing loss, reactive oxygen species, nucleotide-binding oligomerization domain-like receptor protein 3 , inflammasome

\section{Introduction}

Cytomegalovirus (CMV) is the most significant cause of developmental disorders associated with intrauterine infection in humans, potentially resulting in hearing loss (1-3). Only 10-15\% of children with congenital CMV infection exhibit clinical signs at birth, although even children who appear asymptomatic at birth are at risk of neurodevelopmental sequelae $(2,4)$. Sensorineural hearing loss (SNHL) occurs in symptomatic and asymptomatic CMV infections $(5,6)$. It was reported that the progression of CMV brain infection in neonatal mice pivots on innate and adaptive immune responses (7). A previous study by our group indicated that cleaved caspase- 1 and downstream inflammatory factors, including interleukin (IL)-1 $\beta$ and IL-18, were activated in CMV-infected cochleae (8). However, little is known regarding the factors that initiate the inflammatory process in SNHL induced by CMV.

In the inner ear, the spiral ganglion neurons serve the important function of conveying electric signals to the brain (9). Inflammation may also damage the spiral ganglion neurons (SGN) of the inner ear through the round window membrane and cause SNHL (10). In addition, Schachtele et al (1) have reported that cochlear SGN apoptosis occurred in neonatal mice with murine (m) CMV infection, leading to SNHL. However, the mechanisms underlying the induction of this apoptotic process of SGN have remained elusive.

Studies on viral brain infection in adult and neonatal mice suggest that recruitment of neutrophils and macrophages is the primary innate defense mechanism against CMV (11). However, these immune cells may also be harmful to the inner ear by production of reactive oxygen species (ROS) (1). Substantial evidence has suggested that ROS has an important role in the pathogenesis of inflammation and tissue injury $(12,13)$.

Intracellular ROS generation may induce the activation of the nucleotide-binding oligomerization domain-like receptor protein 3 (NLRP3) inflammasome in response to a variety of cellular stressors (14). The NLRP3 inflammasome acts as a molecular platform through inducing the maturation of pro-inflammatory cytokines, including IL-1 $\beta$ and IL-18 $(15,16)$. 
A number of studies have indicated that the NLRP3 inflammasome, which consists of NLRP3, apoptosis-associated speck-like protein containing a carboxy-terminal caspase recruitment domain (ASC) and caspase-1, is associated with cell dysfunction. It has been demonstrated that NLRP3 inflammasome activation is critical for the cell damage under various circumstances (17). However, it has remained elusive whether CMV activates the NLRP3 inflammasome through ROS in the whole inner ear and SGN.

In the present study, a model of hearing loss was established in mice inoculated with murine (M)CMV early in the postnatal period to observe whether the MCMV-induced hearing loss is based on the generation of ROS to induce inflammation in vivo and in cultured SGN. The results indicated that CMV induces SNHL, at least in part, via this mechanism.

\section{Materials and methods}

Reagents. Anti-NLRP3 (1:1,000 dilution; cat. no. 19771-1-AP) antibody was purchased from ProteinTech Group, Inc. (Chicago, IL, USA). Anti-ASC antibody (1:1,000 dilution; cat. no. ST1121) and anti-caspase-1 (p20) antibody (1:1,000 dilution; cat. no. AP1043) were purchased from Chemicon (EMD Millipore, Billerica, MA, USA). Anti- $\beta$-actin antibody (1:10,000 dilution; cat. no. 13E5) was purchased from Cell Signaling Technology, Inc. (Danvers, MA, USA), and pro-IL-18 and IL-18 (1:1,000 dilution; cat. no. BAF-1520) pro-IL-1 $\beta$ and IL-1 $\beta$ (1:1,000 dilution; cat. no. AF-401-SP) antibodies were purchased from R\&D Systems (Minneapolis, MN, USA). Anti-NeuN (1:500 dilution; cat. no. ab177487) and ROS inhibitor N-acetyl-L-cysteine (NAC; 1:1,000 dilution; cat. no. ab143032) were purchased from Abcam (Cambridge, UK). ELISA kits for IL-1 $\beta / 18$ were purchased from USCN Life Sciences, Inc. (Wuhan, China).

Virus and animals. MCMV (Smith strain) was provided by Dr Meng Hong (Medical College of Shandong University, Jinan, China). Specific identification of the virus was described previously (18). MCMV was replicated in NIH3T3 cells (American Type Culture Collection, Manassas, VA, USA) cultured with Dulbecco's modified Eagle's medium (DMEM; Gibco; Thermo Fisher Scientific, Inc., Waltham, MA, USA) containing $10 \%$ fetal bovine serum (FBS; Gibco; Thermo Fisher Scientific, Inc.), streptomycin $(300 \mathrm{~g} / \mathrm{ml})$ and penicillin (300 U/ml). The supernatant of the MCMV-infected NIH3T3 cells was centrifuged at $1,600 \mathrm{x} \mathrm{g}$ for $10 \mathrm{~min}$ at $4^{\circ} \mathrm{C}$. Viral stock titers were determined using $3 \mathrm{~T} 3$ cells as $50 \%$ tissue culture infective doses (2,000 pfu) per milliliter. MCMV was stored as aliquots at $-80^{\circ} \mathrm{C}$ until use.

Murine mouse model. Newborn BALB/c mice (within $24 \mathrm{~h}$ of birth, weight 1.5-2 g, $\mathrm{n}=80$ ) were divided into two groups (each group had 40 mice, 1:1 male to female ratio). MCMV suspension $(50 \%$ tissue culture infective dose $=2,000 \mathrm{pfu} / \mathrm{ml}$, $15 \mathrm{ml}$ ) was slowly injected into the cerebral hemisphere of mice using a micro syringe along the sagittal suture, with a depth of 2-3 mm (19). Following injection, the injected group and the control group mice were separated into two cages and fed by hand to mimic the maternal feeding, and were maintained at room temperature under a $12 \mathrm{~h}$ light/dark cycle and a humidity of 50-60\%. Mice were observed for their growth and developmental status daily (19). Mice were supplied by the Animal Center at Xuzhou Medical University (Xuzhou, China). Animal experiments were performed in accordance with a protocol approved by the Ethics Committee of the Experimental Animal Center at Xuzhou Medical University (Xuzhou, China).

Evaluation of hearing loss. Hearing loss was evaluated at 3 weeks of age by measuring the auditory brainstem responses (ABRs). Mice were anesthetized with $100 \mathrm{mg} / \mathrm{kg}$ ketamine hydrochloride (INN ketamine) and $10 \mathrm{mg} / \mathrm{kg}$ xylazine, and intubated with a 22-gauge catheter, and artificially ventilated (Hallowell EMC, Pittsfield, MA, USA) through an intraperitoneal injection. Click stimuli were generated and ABR waveforms were recorded in decreasing $1 \mathrm{~dB}$ intervals from a $90 \mathrm{~dB}$ sound pressure level until a threshold was reached (no waveforms were visualized).

Primary culture and identification of SGN. Ten neonatal BALB/c mice were immersed in $75 \%$ ethanol after receiving anesthesia by inhalation of ethyl ether. The bilateral temporal bones were removed, and the spiral ganglion tissue was dissected from the stria vascularis and basilar membrane. The tissue was then digested with $0.25 \%$ trypsin (without EDTA) (cat. no. A610609; Sangon Biotech Co., Ltd., Shanghai, China) for $30 \mathrm{~min}$ at $37^{\circ} \mathrm{C}$. After the samples were centrifuged for $8 \mathrm{~min}$ at $1,600 \mathrm{x} \mathrm{g}$, the cells were resuspended and placed in $35-\mathrm{mm}$ cell culture plates $\left(2 \times 10^{4}\right.$ cells $)$ coated with poly-L-lysine. Cells were maintained in DMEM/F12 (GE Healthcare, Little Chalfont, UK), supplemented with $20 \%$ FBS, $10 \%$ neurotrophic factor $\mathrm{B} 27$ and $1 \%$ penicillin-streptomycin, and placed in an incubator with $5 \% \mathrm{CO}_{2}$ and $95 \%$ air at $37^{\circ} \mathrm{C}$. Cytarabine $(5 \mu \mathrm{mol} / \mathrm{ml})$ was used to purify the SGN for $72 \mathrm{~h}$. NF-200 (1:500 dilution; cat. no. ab177487; Abcam) was the primary antibody added at $4^{\circ} \mathrm{C}$ for $24 \mathrm{~h}$, followed by the Alexa Fluor 488 goat anti-mouse $\operatorname{IgG}(\mathrm{H}+\mathrm{L})$ secondary antibody at $37^{\circ} \mathrm{C}$ for $30 \mathrm{~min}$ (1:500 dilution; cat. no. A11032; Thermo Fisher Scientific, Inc.) in order to identify neurons.

\section{Reverse transcription-quantitative polymerase chain reaction} (RT-qPCR). The total RNA of mouse cochlea was extracted using TRIzol reagent according to the manufacturer's protocol (Tiangen Biotech, Beijing, China). Complementary DNA (cDNA) was synthesized from $1 \mathrm{mg}$ total RNA using the ImProm-II ${ }^{\mathrm{TM}}$ Reverse Transcription System (Promega Corp., Madison, WI, USA). PCR amplification of the cDNA and quantification was then performed using SYBR Green (Stratagene; Agilent Technologies, Inc., Santa Clara, CA, USA). The PCR conditions for the Mx3000P QPCR System (Stratagene) were as follows: 40 denaturation cycles at $95^{\circ} \mathrm{C}$ for $10 \mathrm{sec}$, annealing at $60^{\circ} \mathrm{C}$ for $10 \mathrm{sec}$ and elongation at $72^{\circ} \mathrm{C}$ for $10 \mathrm{sec}$. The relative product levels were quantified using the 2(-Delta Delta $C(T)$ ) method (20). Data presented are representative of three independent experiments. $\beta$-actin as internal standard was arbitrarily assigned a value of 1.0. The sequence-specific primers used for qPCR were as follows: IL-18 sense, 5'-CCA AGGAAATCGGCCTCTAT-3' and antisense, 5'-TTGTTC TCACAGGAGAGAGTTGA-3'; IL-1 $\beta$ sense, 5'-AAGAAG AACCCGTCCTCTGCAACA-3' and antisense, 5'-TCAGCT 
CATACGTGCCAGACAACA-3'; $\beta$-actin (control) sense, 5'-GGGTCAGAAGGATTCCTATG-3' and antisense, 5'-GGT CTCAAACATGATCTGGG-3'.

Western blot analysis. Western blot analysis was performed as described previously (21). The cells were solubilized in lysis buffer $(100 \mathrm{mmol} / \mathrm{l}$ Tris- $\mathrm{HCl}, 2 \% \mathrm{SDS}, 10 \%$ glycerin, $100 \mathrm{mmol} / 1 \mathrm{DL}$-dithiothreitol and protease inhibitors; $\mathrm{pH}$ 6.8). Protein extraction of the cytosolic and mitochondrial fractions was performed using a multiple centrifugation method as described previously $(8,21)$. The sample protein concentrations were determined using a BCA protein assay kit (Thermo Fisher Scientific, Inc.). Protein (30 ng) were separated using SDS-PAGE (10\% gel) and then electrotransferred onto a nitrocellulose membrane (EMD Millipore). After blocking for $3 \mathrm{~h}$ with 3\% bovine serum albumin (BSA) (E661003; Sangon Biotech Co., Ltd., Shanghai, China) in Tris-buffered saline with $0.1 \%$ Tween-20 (TBST), membranes were incubated overnight at $4^{\circ} \mathrm{C}$ with primary antibodies in TBST containing 3\% BSA and then fluorescently labeled secondary antibody (1:10,000; cat. no. A7650; Sigma-Aldrich; Merck $\mathrm{KGaA}$ ) for $1 \mathrm{~h}$ at room temperature. After washing, the protein bands were scanned with the Odyssey Infrared Imaging System (Li-Cor Biosciences, Waltham, MA, USA) (without ECL/staining).

Immunofluorescence. Cells were grown in 48-well plates. After the respective treatments, cells were washed twice with PBS and fixed with freshly prepared $4 \%$ paraformaldehyde at room temperature for $15 \mathrm{~min}$. For inner ear tissue staining, inner ear tissue was horizontally sliced into $5-\mu \mathrm{m}$ sections, which were mounted on glass slides. Antigen accessibility was increased by treatment with $2 \%$ Triton $\mathrm{X}-100$ for $10 \mathrm{~min}$ at $37^{\circ} \mathrm{C}$. The samples were then blocked with $3 \%$ BSA for $30 \mathrm{~min}$. The samples and cells were incubated with the primary antibodies overnight at $4^{\circ} \mathrm{C}$. After washing three times with PBS, samples and cells were stained with a secondary antibody Alexa Fluor 488 goat anti-mouse $\operatorname{IgG}(\mathrm{H}+\mathrm{L})$ (1:500 dilution; cat. no. A11032; Thermo Fisher Scientific, Inc.) for $1 \mathrm{~h}$ at $37^{\circ} \mathrm{C}$. The nuclei were counterstained with DAPI for $15 \mathrm{~min}$. After each incubation, the samples and cells were washed thrice with PBS for $5 \mathrm{~min}$ each. The morphology of SGN cells and the inner ear tissues were observed and images were captured with an Olympus DSU-IX81 confocal microscope (Olympus, Tokyo, Japan).

Detection of intracellular ROS. The generation of intracellular ROS was measured by monitoring the increasing fluorescence of 2070-dichlorofluorescein (DCF). 2070-dichlorodihydorofluorescein diacetate (DCFH-DA; Sigma-Aldrich; Merck KGaA, Darmstadt, Germany) is cell-permeant and enters the cells where intracellular esterases cleave off the diacetated group. The resulting DCFH was retained in the cytoplasm and oxidized to DCF by ROS. SGN were seeded into each well of a 48 -well plate. After $24 \mathrm{~h}$ of infection with $10 \mu 1 \mathrm{MCMV} / 1 \mathrm{ml}$ DMEM at 2,000 pfu/ml, cells were then washed once with phenol red-free medium and incubated in $200 \mu \mathrm{l}$ DCFH-DA working solution $(20 \mathrm{mM})$ at $37^{\circ} \mathrm{C}$ for $30 \mathrm{~min}$. The cells were observed under a fluorescence microscope (Olympus). Fluorescence ( $488 \mathrm{~nm}$ excitation and $530 \mathrm{~nm}$ emission) was measured after 30 min using a microplate fluorometer (Thermo Fisher Scientific, Inc.). In order to inhibit the generation of ROS, cells were pretreated with $5 \mathrm{mM}$ NAC (ROS inhibitor) for $2 \mathrm{~h}\left(37^{\circ} \mathrm{C}\right)$ prior to MCMV injection.

Measurement of total superoxide dismutase (T-SOD) and malondialdehyde (MDA). T-SOD and MDA activities were measured using detection kits (KGT00150 and KGT003; Nanjing KeyGen Biotech Co., Ltd., Nanjing, China) according to the manufacturers' protocols. T-SOD activities were examined via the xanthine oxidase method. MDA levels were detected using the 2-thiobarbituric acid method. Colorimetric measurements were preformed using a multi-mode microplate reader (Synergy 2; Bio-Tek, Winooski, VT, USA).

ELISA. The ELISA kits of IL-1 $\beta$ included the pro-forms and IL-18 included the pro-forms were purchased from USCN Life Sciences (cat. nos. SEA563Mu and SEA064Mu). Cell supernatants were collected. For all ELISAs, the substrate solution was tetramethylbenzidine, the reaction was blocked using $2 \mathrm{NH}_{2} \mathrm{SO}_{4}$, and absorbance was read at $450 \mathrm{~nm}$. The assays were carried out according to the manufacturer's protocol. For all assays, Nunc MaxiSorp 96 well ELISA plates were used (cat. no. 439454; Thermo Fisher Scientific, Inc.).

Statistical analysis. Statistical analysis was performed using SPSS 16.0 (SPSS, Inc., Chicago, IL, USA). Values are expressed as the mean \pm standard error of the mean. The data of Fig. 1B were analyzed with the Mann Whitney U test and the data of Figs. 2 and 3 were analyzed by Student's t-test. The data of Fig. 5 were analyzed by one-way analysis of variance followed by Tukey's test. $\mathrm{P}<0.05$ was considered to indicate a statistically significant difference.

\section{Results}

MCMV causes hearing loss in neonatal mouse model. To establish the model of CMV-induced SNHL, neonatal BALB/c mice were injected into the right cerebral lobe with the strain of MCMV (2,000 pfu/ml, $15 \mathrm{ml})$ no later than $24 \mathrm{~h}$ after birth. The results of ABR parameters at 21 days post MCMV infection were identified. As presented in Fig. 1A, a significantly increased ABR threshold was detected in a representative CMV-infected mouse compared with those of a representative control mouse. The ABR thresholds for individual ears in control and MCMV-injected mice are displayed in Fig. 1B. These results revealed that the model of CMV-induced SNHL was successfully established.

MCMV infection increases cochlear pro-inflammatory mediators. To assess the expression of pro-inflammatory mediators during MCMV infection, the protein levels of pro-IL-18/1 $\beta$ and IL-18/1 $\beta$ after MCMV infection were evaluated in mouse inner ears tissues. As presented in Fig. 2A, the protein levels of pro-IL-18/1 $\beta$ were increased in MCMV-infected mice, and the protein levels of IL-18/1 $\beta$ displayed the same trends. Regarding the temporal expression of IL-18/1 $\beta$ mRNA, the mRNA levels of IL18 and IL-1 $\beta$ in mouse inner ear tissues were raised after infection with MCMV (Fig. 2B). The secreted levels of IL-1 $\beta$ and IL-18 in inner ear tissues were also increased (Fig. 2C). 
A

Control

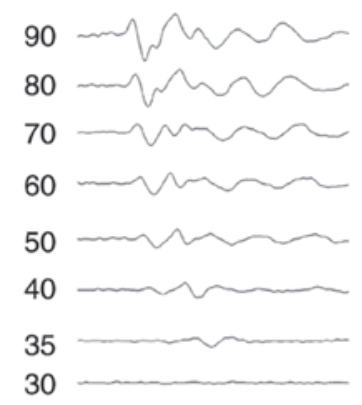

MCMV

90

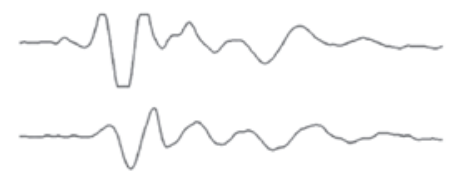

70

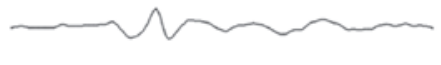

60

55

B

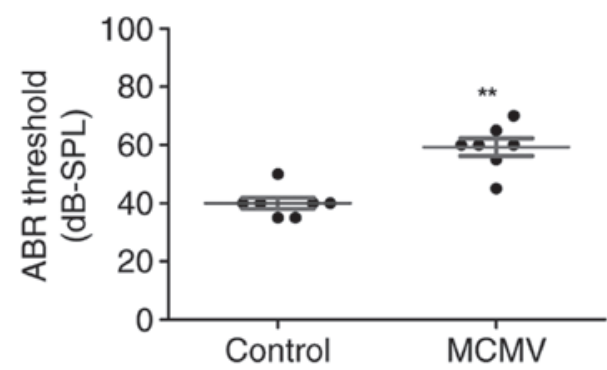

Figure 1. MCMV results in hearing loss in a neonatal mouse model. Mice were subjected to ABR analysis at 21 days of age to determine their hearing ability. (A) Representative curves and (B) quantified ABR in the two groups. MCMV-injected mice exhibited ABR thresholds of -60 dB SPL, which was significantly increased compared with those in control mice $(\mathrm{P}<0.01)$. ABR click stimuli were provided from $90 \mathrm{~dB}$ SPL to $0 \mathrm{~dB}$ in descending-10-dB intervals, and responses to 2,000 sweeps were averaged at each intensity level. Values are expressed as the mean \pm standard deviation. ${ }^{* *} \mathrm{P}<0.01 \mathrm{vs}$. control group. $\mathrm{MCMV}$, murine congenital cytomegalovirus; ABR, auditory brainstem response; SPL, sound pressure level.

A

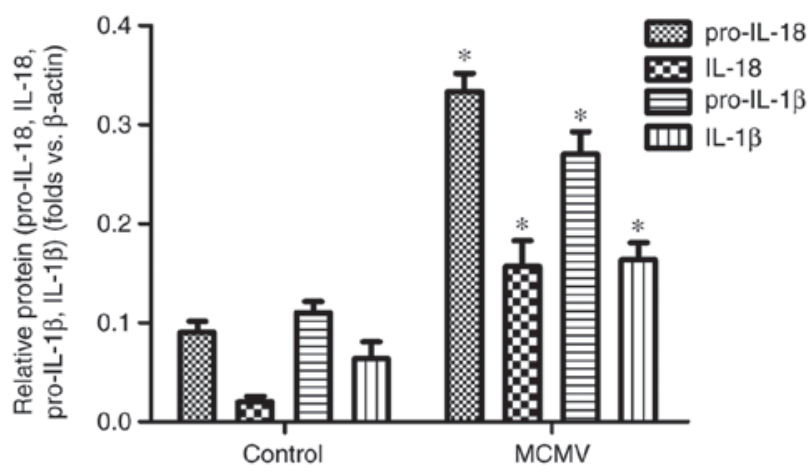

B

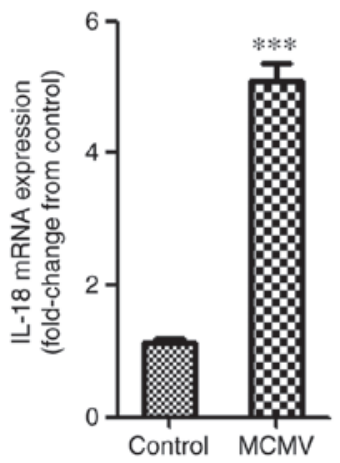

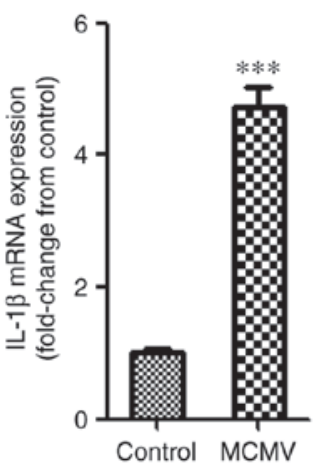

C

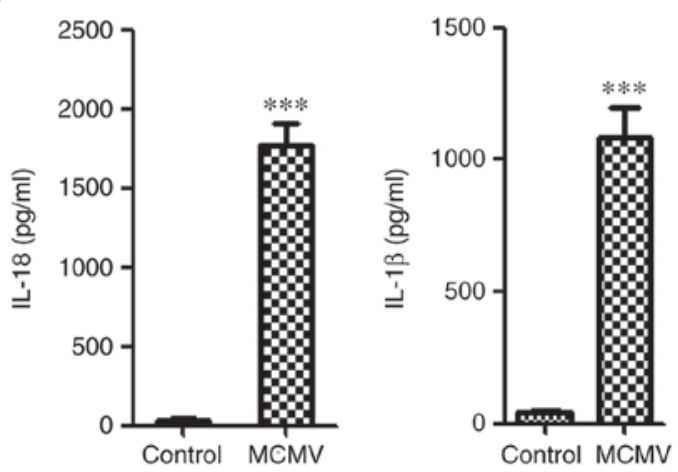

Figure 2. MCMV infection increases cochlear pro-inflammatory mediators in mice. (A) Western blot analysis of the expression of pro-IL18/1 $\beta$ and IL-1 $1 \beta / 18$. (B) IL-18/1 $\beta$ mRNA expression was examined by reverse transcription-quantitative polymerase chain reaction analysis; the expression levels relative to those of $\beta$-actin were normalized to the control group. (C) Protein levels of IL-1 $\beta$ and IL-18 determined by ELISA. Values are expressed as the mean \pm standard deviation ( $\mathrm{n}=3) .{ }^{*} \mathrm{P}<0.05 ;{ }^{* * *} \mathrm{P}<0.001$ vs. the control group. MCMV, murine congenital cytomegalovirus; IL, interleukin.

MCMV induces NLRP3 inflammasome activation in cochlea. To assess whether MCMV induces NLRP3 inflammasome activation in the cochlea, the neonatal $\mathrm{BALB} / \mathrm{c}$ mice were infected with MCMV and after 3 weeks, western blot analysis was performed to assess the protein levels of NLRP3, ASC and caspase-1 (P20). As indicated in Fig. 3A, NLRP3 and 
A

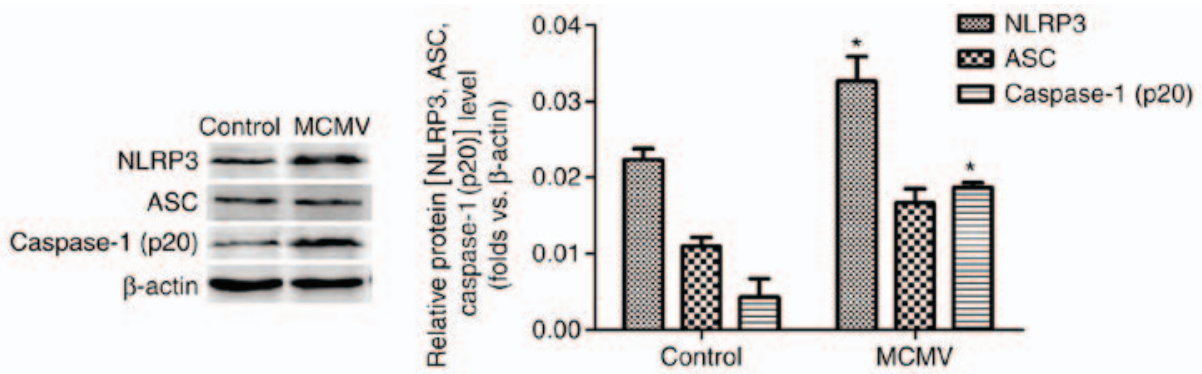

B

B DAP

DAPI

NLRP3
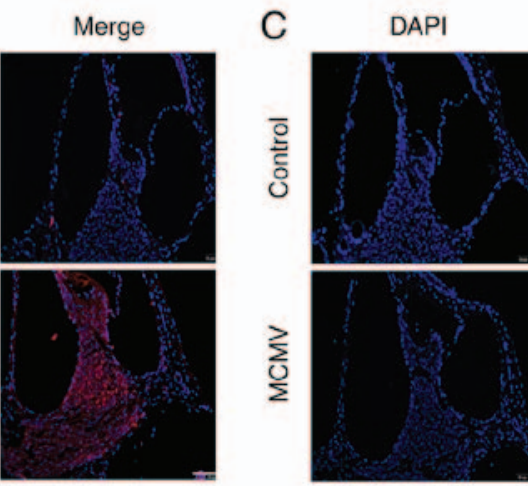

Caspase-1

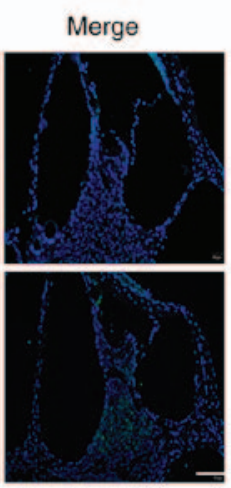

Figure 3. MCMV induces NLRP3 inflammasome activation in the cochlea of mice. (A) Western blot analysis was applied to assess the effect of MCMV infection on the expression levels of NLRP3 inflammasome proteins. Values are expressed as the mean \pm standard deviation $(\mathrm{n}=3)$. ${ }^{*} \mathrm{P}<0.05$ vs. the control group. (B and C) Immunofluorescence analysis of (B) NLRP3 and (C) caspase-1 (p20) in cochlea (scale bar, $50 \mu \mathrm{m}$ ). MCMV, murine congenital cytomegalovirus; NLRP3, nucleotide-binding oligomerization domain-like receptor protein 3.
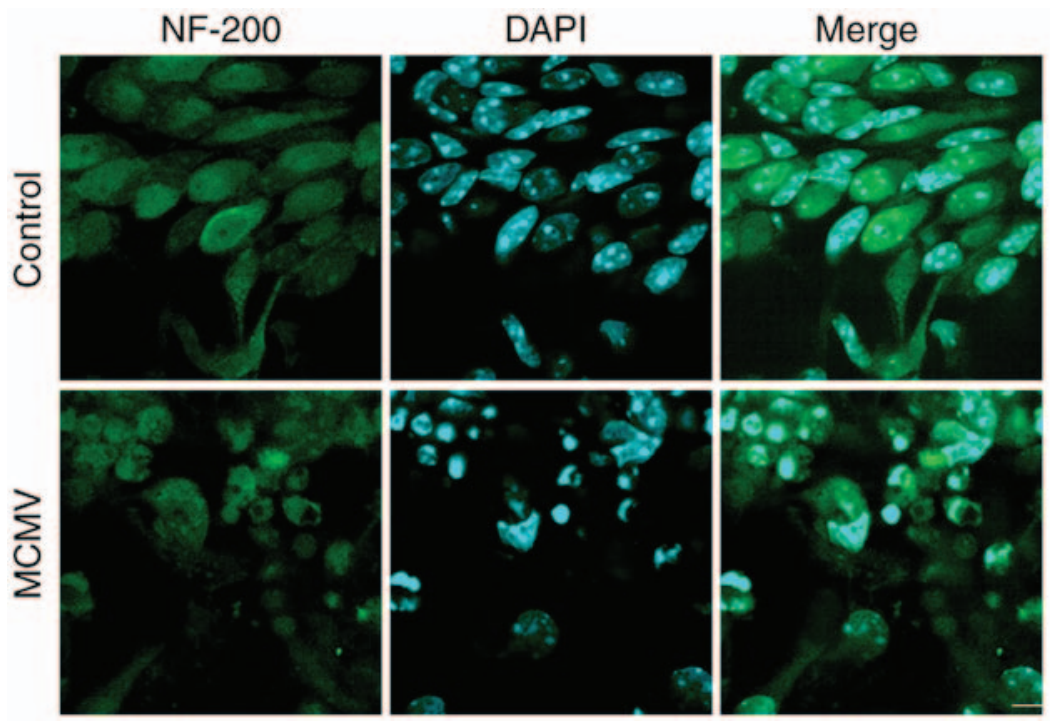

Figure 4. MCMV induces apoptosis of cultured SGN in vitro. Representative confocal microscopy images of confluent cultures of SGN treated with vehicle (control) or MCMV for $24 \mathrm{~h}$, followed by staining for neurons (NF-200; green) and nuclei (DAPI, blue) (scale bar, $50 \mu \mathrm{m}$ ). MCMV, murine congenital cytomegalovirus; SGN, spiral ganglion neurons; NF, neurofilament.

caspase-1 (p20) were increased in cochlea after MCMV infection. However, MCMV had no impact on the level of ASC protein. Immunofluorescence analysis of NLRP3 and caspase-1 (p20) confirmed these outcomes (Fig. 3B and C). These results revealed that the activity of NLRP 3 inflammasome in cochlea was induced after infection with MCMV. MCMV also damaged cultured SGN in vitro. SGN cells have important roles in processes associated with neurotransmitters. To investigate whether MCMV damages SGN cells, these cells were cultured in vitro and infected with MCMV for
$24 \mathrm{~h}$, following which SGN was marked by NF-200 antibody and cells were counterstained with DAPI. As presented in Fig. 4, MCMV induced and shrinkage of cells in SGN cells in contrast to the control group. These results suggested that MCMV damages the cultured SGN in vitro.

$C M V$ induces the generation of ROS in cultured SGN in vitro. Prolonged exposure to excessive ROS has been implicated in neurotoxicity during viral brain infection (22). To investigate whether MCMV induced ROS production by SGN in vitro, the 
A
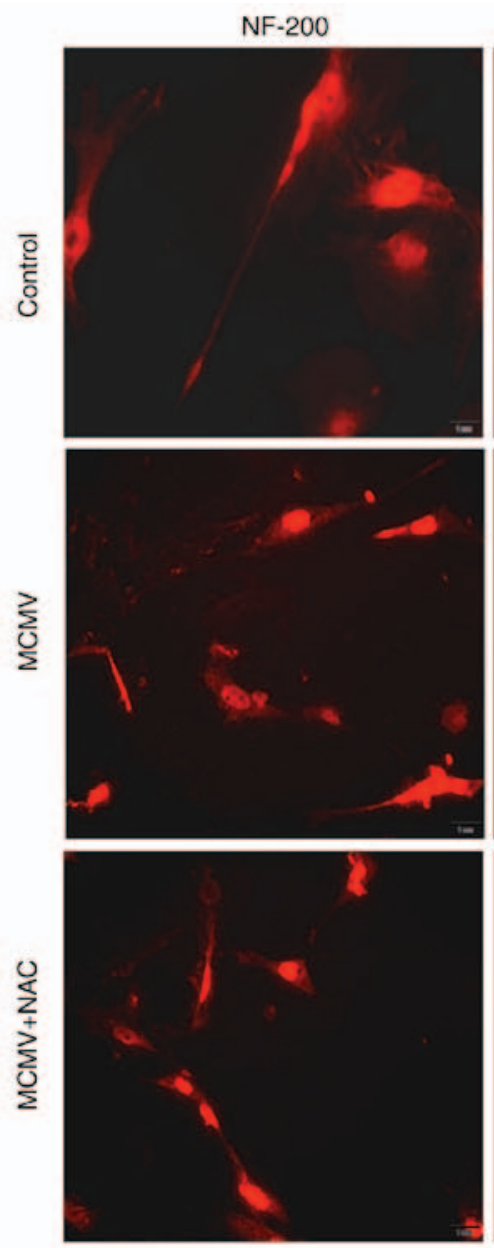

B

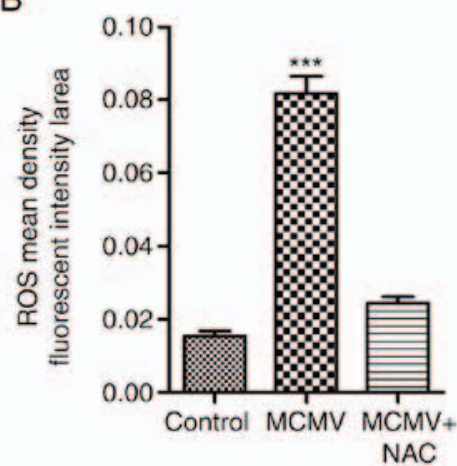

ROS
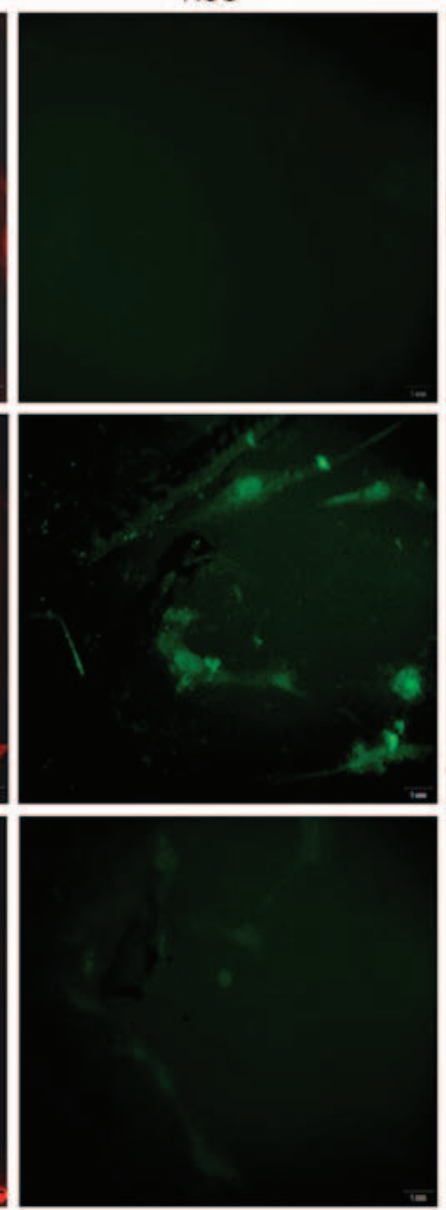
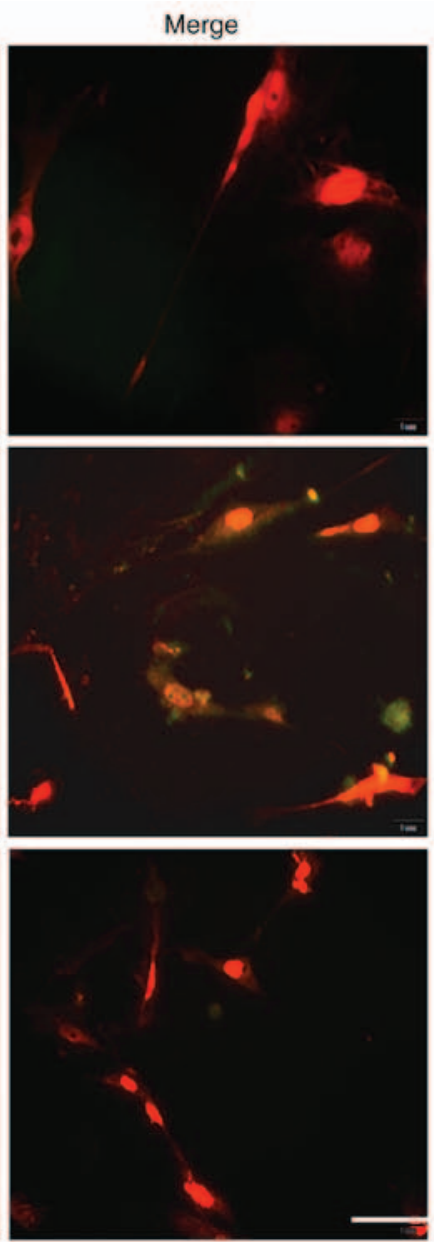

C

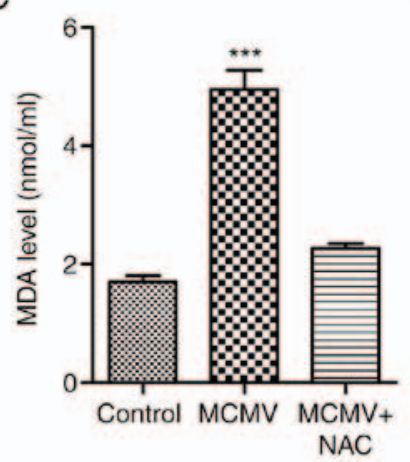

D

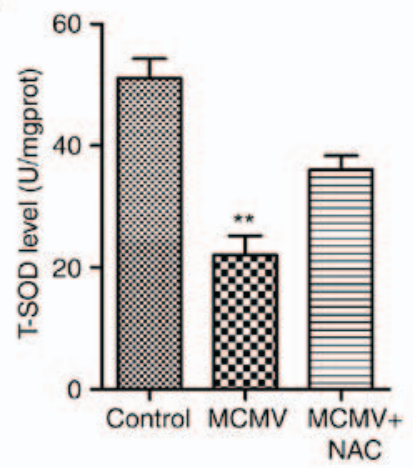

Figure 5. CMV-induced ROS expression in cultured SGN. (A) ROS levels were measured with the dichlorodihydorofluorescein diacetate fluorescence probe (green). Immunohistochemical staining for NF-200 (red), a marker for SGN, was also performed. ROS inhibitor NAC decreased the ROS levels (scale bar, $50 \mu \mathrm{m}$ ). (B) Mean fluorescent intensity of ROS was determined from A. (C) MDA levels were examined with the 2-thiobarbituric acid method. (D) T-SOD activities were examined via the xanthine oxidase method. ${ }^{* *} \mathrm{P}<0.01 ;{ }^{* * *} \mathrm{P}<0.001$ vs. the control and MCMV+NAC groups. Values are expressed as the mean \pm standard deviation. NF, neurofilament; MCMV, murine congenital cytomegalovirus; SGN, spiral ganglion neurons; ROS, reactive oxygen species; MDA, malondialdehyde; NAC, N-acetyl-L-cysteine; T-SOD, total superoxide dismutase.

DCFH-DA assay was employed to determine the level of ROS. As presented in Fig. 5A and B, the cellular fluorescence intensity of the metabolite DCF was increased to multiple folds of the level in the control group, which was inhibited in the presence of the ROS inhibitor NAC. MDA was also changed in a similar manner to ROS (Fig. 5C). As expected, T-SOD activity displayed an opposite trend (Fig. 5D). These results suggested that CMV increased the level of ROS and MDA in the cultured SGN.
MCMV induces NLRP3 inflammasome activation in cultured $S G N$. The involvement of the NLRP3 inflammasome pathway in cultured SGN was then verified. The expression of NLRP3 and caspase-1 (P20) was detected by immunofluorescence. Immunofluorescence staining for the NLRP3 inflammasome was apparent in the cultured SGN infected with MCMV, while virtually no staining was observed in the control group, and the inflammatory effect of MCMV was reduced when the ROS inhibitor NAC was applied (Fig. 6A). The expression of 


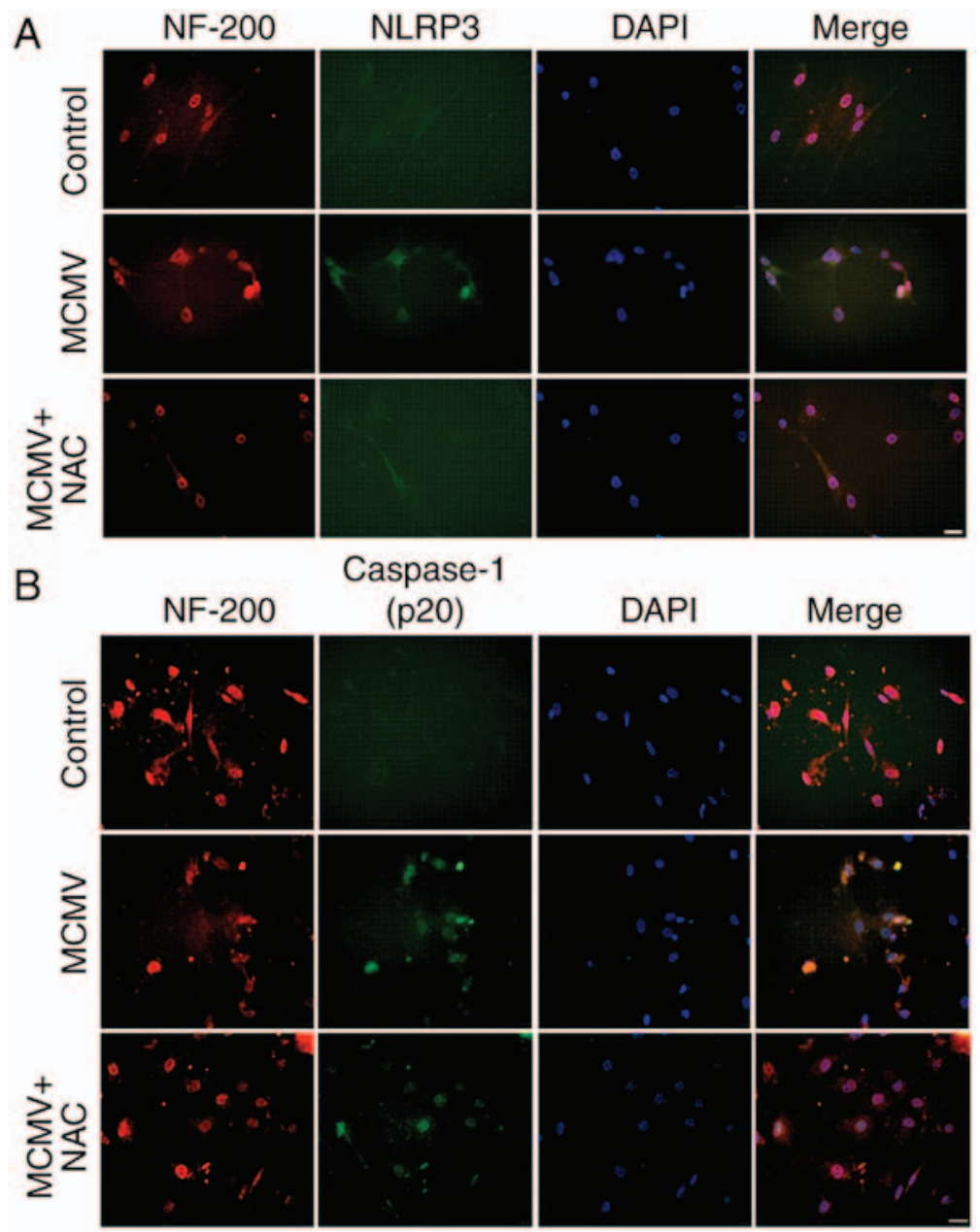

Figure 6. MCMV induces NLRP3 inflammasome activation in cultured SGN. (A) Immunofluorescence analysis of NLRP3 in cultured SGN; immunohistochemical staining for NF-200 (red) as a marker for SGN and NLRP3 (green). (B) Immunofluorescence analysis of caspase-1 (p20) in cultured SGN; immunohistochemical staining for NF-200 (red) and caspase-1 (green). Nuclear counterstain was performed with DAPI (blue; scale bar, $50 \mu \mathrm{m}$ ). SGN, spiral ganglion neurons; NLRP3, nucleotide-binding oligomerization domain-like receptor protein 3; MCMV, murine congenital cytomegalovirus; NeuN, neuronal nuclei; NF, neurofilament; NAC, N-acetyl-L-cysteine.

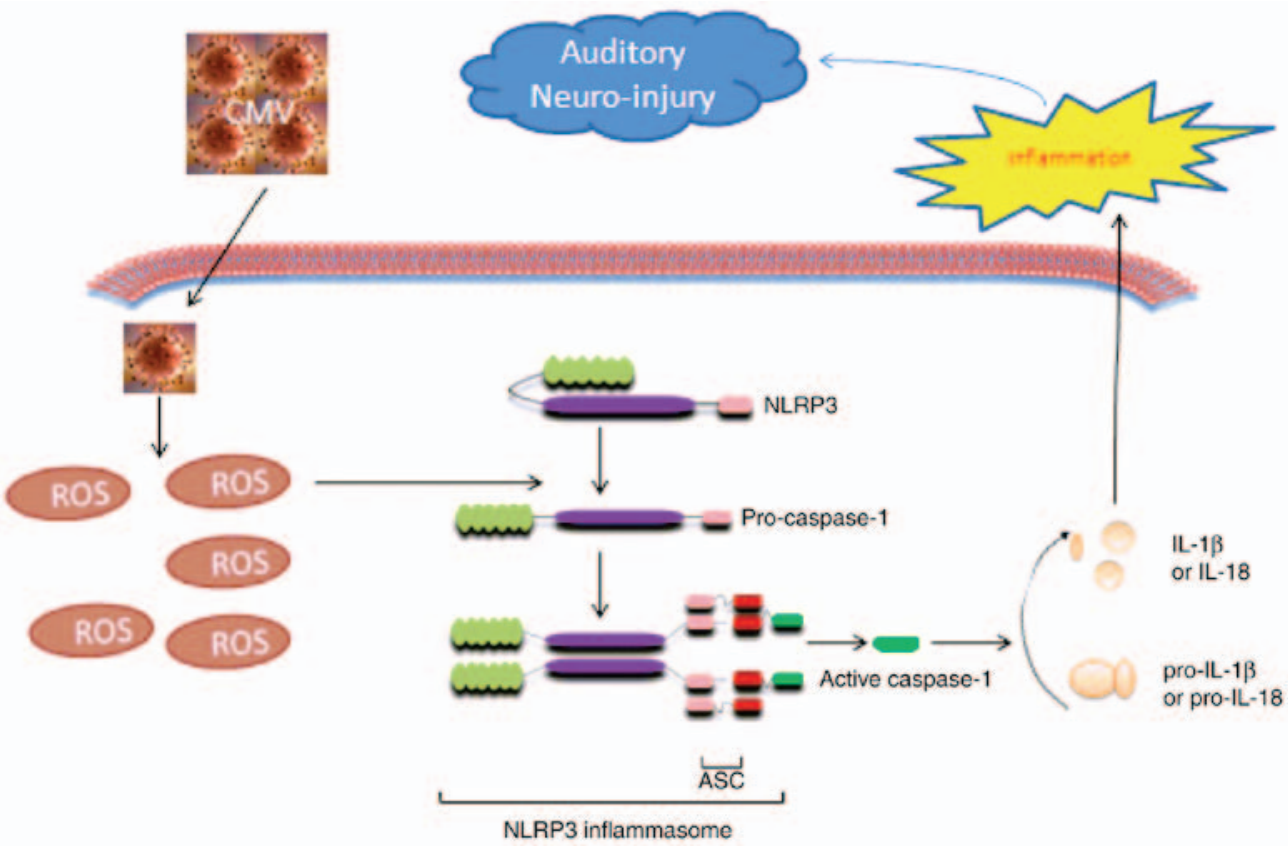

Figure 7. Mechanisms for MCMV-induced sensorineural hearing loss via ROS-mediated inflammation. MCMV increases the level of ROS and activates NLRP3 inflammasome in the cochlea and in cultured spiral ganglion neurons, resulting in caspase-1 activation and increases in IL- $1 \beta$ and IL-18 maturation and release. IL, interleukin; MCMV, murine congenital cytomegalovirus; NLRP3, nucleotide-binding oligomerization domain-like receptor protein 3; ASC, apoptosis-associated speck-like protein containing a carboxy-terminal caspase recruitment domain. 
caspase-1 (P20) displayed the same tendency (Fig. 6B). These results clearly indicated that MCMV markedly increases the expression of NLRP3 and caspase-1 (p20) in the cultured SGN.

\section{Discussion}

To the best of our knowledge, the present study demonstrated that MCMV actives inflammation in vivo and in cultured SGN cells. The possible effects of MCMV may be due to the activation of ROS (Fig. 7) as MCMV increased the level of ROS and activated NLRP3 inflammasome in the cochlea and cultured spiral ganglion neurons, resulting in caspase-1 activation and an increase in IL-1 $\beta$ and IL-18 maturation and release. These results suggested that ROS-induced inflammation via the NLRP3 inflammasome is a mechanism of MCMV-associated SNHL.

The inflammasome is multiprotein complex localized within the cytoplasm of the cell and is responsible for the maturation of pro-inflammatory cytokines, including IL-1 $\beta$ and IL-18, as well as the mediation of a highly inflammatory form of cell death (23). The canonical inflammasome includes NLR (including NLRP1, NLRP3 and NLRC4) and non-NLR (e.g. AIM2) types, which is mediated by activation of caspase-1 in response to pathogen-associated molecular patterns and damage-associated molecular pattern molecules $(24,25)$. Sester et al (26) reported that AIM2 antagonist protein p202 had a high expression and low IL-1 $\beta$ output in response to transfected DNA and mouse CMV infection in an autoimmune hemolytic anemia model. Rathinam et al (27) reported that AIM2 binds to cytosolic DNA and initiates inflammasome responses in response to viruses including MCMV and vaccinia, as well as the cytosolic bacterium Francisella tularensis. The results of a previous study by our group are consistent with these previous studies (8). The NLRP3 inflammasome has been implicated in the pathogenesis of a wide variety of diseases, including genetically inherited autoinflammatory conditions, as well as chronic diseases, in which NLRP3 is abnormally activated (28). However, it has remained elusive whether MCMV exerts its effects by affecting the NLRP3 inflammasome in mouse cochlea. The present study demonstrated that MCMV stimulated NLRP3 activity in mouse cochlea. In combination with a previous study by our group, the conclusion was drawn that the effects of MCMV are not only dependent on AIM2, but also NLRP3.

As is well known, the overproduction of ROS causes severe damage to cellular macromolecules and ROS production is an important intracellular inducer of autophagy (9). An accumulating body of evidence has also indicated that ROS has an important role in CMV infection. Xiao et al (29) described that patients infected with human CMV experience an imbalance of redox homeostasis that causes accumulation of ROS at the cellular level. The present study proved that MCMV stimulated ROS activity in mouse cochlea. In addition, MCMV promoted the production of ROS through decreasing SOD activities. This is the underlying mechanism for MCMV-induced SNHL.

Furthermore, the present study proved for the first time, to the best of our knowledge, that MCMV infection increases the level of ROS and activates the NLRP3 inflammasome in cultured SGN in vitro. During the hearing process, SGN receive an electrical signal input from cochlear hair cells and project from the cochlea to the cochlear nucleus; subsequently, the electrical signals are transmitted to the auditory cortex (30). Therefore, the SGN are referred to as the first level of neurons of the auditory system. The dysfunction of SGN often leads to SNHL and causes implantable hearing device failure (9). Esperanza et al (31) suggested that the expression of pro-inflammatory cytokines promotes intra-cochlear fibrosis, as well as loss of the auditory hair cells and SGN. The results of the present study indicated that MCMV triggers ROS-induced inflammation in cultured SGN. This may be the mechanism to explain the SGN loss in SNHL caused by MCMV. The underlying mechanisms require to be further assessed in future studies.

In conclusion, the present study indicated that MCMV activates the NLRP3 inflammasome via production of ROS in mouse cochleae and in cultured SGN. Therefore, ROS-induced NLRP3 inflammasome activation is potentially a novel target for the prevention and treatment of CMV-associated SNHL.

\section{Acknowledgements}

The authors would like to express their sincere gratitude to Dr Meng Hong (Medical College of Shandong University, Jinan, China) for providing MCMV (Smith strain).

\section{Funding}

This study was supported by the National Natural Science Foundation of China (grant nos. 81250042, 81470684 and 81270173) and the Postdoctoral Science Foundation of China (grant no. 2015M571818).

\section{Availability of data and material}

The datasets used and/or analyzed during the current study are available from the corresponding author on reasonable request.

\section{Authors' contributions}

All the authors conceived and designed the study protocol. $\mathrm{HD}, \mathrm{YQ}, \mathrm{WZ}, \mathrm{CW}$ and XS conceived and designed the experiments. SQ, SZ, MC, WJ and BX performed the experiments. WJ, BX and MC analyzed the data. SQ, SZ, MC, HD and YQ provided reagents/materials/analysis tools. WZ, XS, HD, WJ and YQ wrote the paper. All the authors gave final approval of the version to be submitted.

\section{Ethics approval and consent to participate}

Animal experiments were performed in accordance with a protocol approved by the Ethics Committee of the Experimental Animal Center at Xuzhou Medical University (Xuzhou, China).

\section{Consent for publication}

Not applicable. 


\section{Competing interests}

The authors declare that they have no competing interests.

\section{References}

1. Schachtele SJ, Mutnal MB, Schleiss MR and Lokensgard JR: Cytomegalovirus-induced sensorineural hearing loss with persistent cochlear inflammation in neonatal mice. J Neurovirol 17 201-211, 2011.

2. Cheeran MC,Lokensgard JR and Schleiss MR: Neuropathogenesis of congenital cytomegalovirus infection: Disease mechanisms and prospects for intervention. Clin Microbiol Rev 22: 99-126, 2009.

3. Li L, Kosugi I, Han GP, Kawasaki H, Arai Y, Takeshita T and Tsutsui Y: Induction of cytomegalovirus-infected labyrinthitis in newborn mice by lipopolysaccharide: A model for hearing loss in congenital CMV infection. Lab Invest 88: 722-730, 2008.

4. Grosse SD, Ross DS and Dollard SC: Congenital cytomegalovirus (CMV) infection as a cause of permanent bilateral hearing loss: A quantitative assessment. J Clin Virol 41: 57-62, 2008.

5. Ikuta K, Ogawa H, Hashimoto H, Okano W, Tani A, Sato E Kosugi I, Kobayashi T, Omori K and Suzutani T: Restricted infection of murine cytomegalovirus (MCMV) in neonatal mice with MCMV-induced sensorineural hearing loss. J Clin Virol 69: 138-145, 2015.

6. Wang Y, Patel R, Ren C, Taggart MG, Firpo MA, Schleiss MR and Park AH: A comparison of different murine models for cytomegalovirus-induced sensorineural hearing loss. Laryngoscope 123: 2801-2806, 2013

7. Bantug GR, Cekinovic D, Bradford R, Koontz T, Jonjic S and Britt WJ: CD8 ${ }^{+} \mathrm{T}$ lymphocytes control murine cytomegalovirus replication in the central nervous system of newborn animals. J Immunol 181: 2111-2123, 2008.

8. Xi S, Yanfen D, Ya L, Zenlu Z, Huan L, Shiwei Q, Yaohan L, Weiwei $\mathrm{G}$ and Yuehua Q: Inflammasome activation in mouse inner ear in response to MCMV induced hearing loss. J Otol 10: 143-149, 2015.

9. Zuo WQ, Hu YJ, Yang Y, Zhao XY, Zhang YY, Kong W and Kong WJ: Sensitivity of spiral ganglion neurons to damage caused by mobile phone electromagnetic radiation will increase in lipopolysaccharide-induced inflammation in vitro model. J Neuroinflammation 12: 105, 2015

10. Juhn SK, Jung MK, Hoffman MD, Drew BR, Preciado DA, Sausen NJ, Jung TT, Kim BH, Park SY, Lin J, et al: The role of inflammatory mediators in the pathogenesis of otitis media and sequelae. Clin Exp Otorhinolaryngol 1: 117-138, 2008.

11. Nesin $\mathrm{M}$ and Cunningham-Rundles S: Cytokines and neonates. Am J Perinatol 17: 393-404, 2000.

12. Riedl MA and Nel AE: Importance of oxidative stress in the pathogenesis and treatment of asthma. Curr Opin Allergy Clin Immunol 8: 49-56, 2008.

13. Ciencewicki J, Trivedi S and Kleeberger SR: Oxidants and the pathogenesis of lung diseases. J Allergy Clin Immunol 122: 456-470, 2008

14. Zhou R, Yazdi AS, Menu P and Tschopp J: A role for mitochondria in NLRP3 inflammasome activation. Nature 469: 221-225, 2011.

15. Schroder K, Zhou R and Tschopp J: The NLRP3 inflammasome: A sensor for metabolic danger? Science 327: 296-300, 2010.

16. Schroder K and Tschopp J: The inflammasomes. Cell 140: 821-832, 2010.
17. Martinon F, Pétrilli V, Mayor A, Tardivel A and Tschopp J: Gout-associated uric acid crystals activate the NALP3 inflammasome. Nature 440: 237-241, 2006.

18. Stinski MF: Human cytomegalovirus: Glycoproteins associated with virions and dense bodies. J Virol 19: 594-609, 1976.

19. Li X, Shi X, Wang C, Niu H, Zeng L and Qiao Y: Cochlear Spiral ganglion neuron apoptosis in neonatal mice with murine cytomegalovirus-induced sensorineural hearing loss. J Am Acad Audiol 27: 345-353, 2016.

20. Livak KJ and Schmittgen TD: Analysis of relative gene expression data using real-time quantitative PCR and the $2^{-\Delta \Delta C_{\mathrm{T}}}$ method. Methods 25: 402-408, 2001.

21. Shi X, Tian B, Ma C, Liu L, Zhang N, Na Y, Li J, Lu J and Qiao Y: GSK3 $\beta$ activity is essential for senescence-associated heterochromatin foci (SAHF) formation induced by HMGA2 in WI38 cells. Am J Transl Res 9: 167-174, 2017.

22. Schachtele SJ, Hu S, Little MR and Lokensgard JR: Herpes simplex virus induces neural oxidative damage via microglial cell Toll-like receptor-2. J Neuroinflammation 7: 35, 2010.

23. Luo B, Li B, Wang W, Liu X, Xia Y, Zhang C, Zhang M, Zhang Y and An F: NLRP3 gene silencing ameliorates diabetic cardiomyopathy in a type 2 diabetes rat model. PLoS One 9: e104771, 2014.

24. Xie M, Yu Y, Kang R, Zhu S, Yang L, Zeng L, Sun X, Yang M, Billiar TR, Wang H, et al: PKM2-dependent glycolysis promotes NLRP3 and AIM2 inflammasome activation. Nat Commun 7: 13280, 2016.

25. Zorman J, Susjan P and Hafner-Bratkovic I: Shikonin suppresses NLRP3 and AIM2 inflammasomes by direct inhibition of caspase-1. PLoS One 11: e0159826, 2016.

26. Sester DP, Sagulenko V, Thygesen SJ, Cridland JA, Loi YS, Cridland SO, Masters SL, Genske U, Hornung V, Andoniou CE, et al: Deficient NLRP3 and AIM2 inflammasome function in autoimmune NZB mice. J Immunol 195: 1233-1241, 2015.

27. Rathinam VA, Jiang ZZ, Waggoner SN, Sharma S, Cole LE, Waggoner L, Vanaja SK, Monks BG, Ganesan S, Latz E, et al: The AIM2 inflammasome is essential for host defense against cytosolic bacteria and DNA viruses. Nat Immunol 11: 395-403, 2010.

28. Abderrazak A, Syrovets T, Couchie D, El Hadri K, Friguet B, Simmet $\mathrm{T}$ and Rouis M: NLRP3 inflammasome: From a danger signal sensor to a regulatory node of oxidative stress and inflammatory diseases. Redox Biol 4: 296-307, 2015.

29. Xiao J, Deng J, Lv L, Kang Q, Ma P, Yan F, Song X, Gao B, Zhang $\mathrm{Y}$ and $\mathrm{Xu} \mathrm{J}$ : Hydrogen peroxide induce human cytomegalovirus replication through the activation of p38-MAPK signaling pathway. Viruses 7: 2816-2833, 2015.

30. Bailey EM and Green SH: Postnatal expression of neurotrophic factors accessible to spiral ganglion neurons in the auditory system of adult hearing and deafened rats. J Neurosci 34: 13110-13126, 2014.

31. Bas E, Goncalves S, Adams M, Dinh CT, Bas JM, Van De WaterTR and Eshraghi AA: Spiral ganglion cells and macrophages initiate neuro-inflammation and scarring following cochlear implantation. Front Cell Neurosci 9: 303, 2015.

This work is licensed under a Creative Commons Attribution-NonCommercial-NoDerivatives 4.0 International (CC BY-NC-ND 4.0) License. 